\title{
Erratum: supersymmetric completion of Gauss-Bonnet combination in five dimensions
}

\section{Mehmet Ozkan and Yi Pang}

George P. ES Cynthia Woods Mitchell Institute for Fundamental Physics and Astronomy, Texas A\&M University, College Station, TX 77843, U.S.A.

E-mail: mozkan@tamu.edu, pangyi1@physics.tamu.edu

ERRATUM TO: JHEP03(2013)158

ARXIV EPRINT: 1301.6622

- In eq. (3.5), the coefficient of $N^{2} L^{-1}$ should be + instead of -.

- In eq. (4.4), the coefficient of $N^{2} L^{-1}$ should be - instead of + .

- In eq. (4.18), the torsionful covariant derivative acting on $G_{a b}$ should be changed to $\nabla_{\mu}(\omega+)$ form $D_{\mu}(\omega+)$.

- In eq. (5.22), the coefficient of $N^{2} L^{-1}$ should be - instead of + . 